Revue européenne des sciences sociales

European Journal of Social Sciences

La sociologie en quête d'une théorie générale

\title{
Pour une sociologie des formes
}

Jacques Coenen-Huther

\section{OpenEdition}

\section{Journals}

Édition électronique

URL : http://journals.openedition.org/ress/169

DOI : $10.4000 /$ ress. 169

ISSN : 1663-4446

\section{Éditeur}

Librairie Droz

Édition imprimée

Date de publication : 1 février 2008

Pagination : 67-83

ISBN : 978-2-600-01221-8

ISSN : 0048-8046

Référence électronique

Jacques Coenen-Huther, "Pour une sociologie des formes », Revue européenne des sciences sociales [En ligne], XLVI-140 | 2008, mis en ligne le 01 février 2011, consulté le 03 mai 2019. URL : http:// journals.openedition.org/ress/169; DOI : 10.4000/ress.169 


\section{Jacques COENEN-HUTHER}

\section{POUR UNE SOCIOLOGIE DES FORMES}

Les chercheurs de terrain expérimentés savent que le moyen le plus sûr de faire progresser la connaissance sociologique est d'élargir le cadre de l'étude monographique et de passer du particulier au général pour pouvoir effectuer l'opération inverse ultérieurement. Une recherche menée dans un village conduira, par exemple, à l'étude des modes de sociabilité en milieu rural. Un pas de plus dans la voie de la généralisation permettra de définir le milieu rural comme un contexte social caractérisé par la confusion des rôles, à l'inverse de la société urbaine ${ }^{1}$. A ce niveau de généralité et d'abstraction, il deviendra possible de mettre en rapport l'agencement des rôles avec les modalités du contrôle social. Contrairement à la société villageoise où la superposition des rôles permet un contrôle social très direct et parfois très pesant, le milieu de la grande ville, caractérisé par la ségrégation des rôles et la segmentation du social, crée les conditions structurelles de l'individualisme moderne. C'est une démarche comparative qui rend possible ce passage d'un niveau d'analyse à l'autre. Celle-ci exige en préalable un appareil conceptuel - contexte d'interaction, système de rôles, réseau de relations - pouvant offrir des critères de comparaison portant sur les éléments formels des relations. Glaser et Strauss parlent à ce sujet d' "analyse comparative constante », permettant de passer d'une théorie substantielle (substantive theory), fondée sur la recherche dans un domaine particulier, à une théorie formelle (formal theory), reliant plusieurs domaines d'investigation par les aspects formels des phénomènes étudiés $(1967,1973$, pp. 79-88). Ces auteurs illustrent le mécanisme d'alternance des théories substantielles et formelles par un exemple issu de leur recherche publiée sous le titre Awareness of Dying (1965). Le patient en phase terminale étant défini comme un individu affecté d'un changement de statut non programmé (a non scheduled status passage), il devient possible d'utiliser la théorie «substantielle» portant sur les «contextes de conscience» de la mort prochaine, comme point de départ (springboard or stepping stone) d'une théorie «formelle» ayant trait aux statuts, différences de statuts et modifications de statuts (Glaser et Strauss, 1967, 1973, pp. 33 et 79). On trouve la même stratégie d'élaboration d'une théorie formelle chez Howard Becker lorsqu'il extrait une théorie du contrôle social et de la déviance d'observations sur les musiciens de danse et les consommateurs de marijuana (1963).

1 Je me permets de renvoyer à ce sujet à mon article Heurs et malheurs du concept de rôle social (Coenen-Huther, 2005b, pp. 65-82) 
Ces quelques exemples nous suggèrent que la notion de forme peut être liée à des processus - le changement de statut, la déviance - autant qu'à des situations relativement stables. Et de fait, d'autres recherches ont permis de mettre en évidence des processus dont la forme est indépendante de l'objet spécifique. Ainsi en est-il du modèle dit «épidémiologique ${ }^{2}$. Comme l'évolution d'une épidémie dans une population, la diffusion d'une innovation (médicament, objet à usage domestique), la propagation d'une idéologie, la conquête du pouvoir par un mouvement politique, constituent des processus qui présentent globalement la forme d'une courbe logistique, c'est-à-dire d'une courbe en $\mathrm{S}$, plus ou moins étirée ou resserrée selon le rythme de leur déroulement. Ce profil indique que différentes catégories d'une population ne réagissent pas de la même façon au phénomène en question. Dans le cas contraire, la courbe logistique ferait place à une droite d'inclinaison variable. L'allure du processus traduit l'entrée en scène successive de plusieurs catégories d'individus qui adoptent des comportements obéissant à des stimulations ou des contraintes d'ordre psychosocial différentes. Sous les différents cas de figure - adoption graduelle d'un médicament, d'un lavevaisselle ou d'une vision du monde - il y a un mécanisme abstrait de réactivité différentielle qui donne sa forme à la courbe.

Qu'il y ait des phénomènes sociaux présentant les mêmes caractéristiques formelles indépendamment de l'objet spécifique des interactions concernées, notre expérience quotidienne nous permet aisément de nous en rendre compte. Le changement de taille d'un groupe, la croissance d'une entreprise, le gonflement d'une administration, la complexification d'une organisation, l'urbanisation d'une campagne, la diversification d'une population, sont autant de processus dont nous pouvons anticiper plus ou moins les conséquences, abstraction faite de leur contenu particulier. Et ce sont nos attentes plus ou moins précises qui nous font parfois dire que, décidément, «les mêmes causes produisent les mêmes effets » quels que soient les individus impliqués, leurs intentions et leurs espoirs. Sur cette base se développe une sorte de sociologie spontanée, faite de scepticisme et de bon sens, qui préfigure des théories plus élaborées comme, par exemple, la théorie de la reproduction des élites ou celle de la frustration relative. Cette sagesse sociologique préscientifique n'a pas toujours bonne presse car elle détruit bien des illusions. Elle nous suggère que la diversité culturelle ne va pas sans heurts, que les opprimés d'hier peuvent être les oppresseurs d'aujourd'hui, qu'il n'y a pas de guerres propres, que nos alliés peuvent se comporter de façon aussi barbare que nos ennemis, etc.

Les enseignements de la recherche empirique tout comme les leçons de la vie nous amènent à découvrir des constantes à caractère formel que l'analyse peut détacher du cadre particulier de telle ou telle situation d'interaction. Dès lors, il paraît opportun de consacrer davantage d'efforts et de ressources à la mise en évidence d'invariants du comportement humain qui transcendent les époques et les cultures. Un tel programme de recherche conférerait à l'investigation sociologique ce qui lui manque cruellement: la stabilité nécessaire à la cumulativité des connaissances (Coenen-Huther, 2005a). Il favoriserait l'usage de propositions

2 Sur ce point, on consultera utilement l'ouvrage d'Henri Mendras et Michel Forsé, Le changement social. Tendances et paradigmes, Paris, Armand Colin, 1983. 
structurelles ou conditionnelles du type « Si A, alors B». La sociologie se rapprocherait ainsi du statut de discipline scientifique dont elle ne bénéficie en ce moment «qu'en vertu d'une fiction sémantique et d'une espérance philosophique à laquelle les confirmations manquent encore (Lévi-Strauss, 1973, p. 341). Car la recherche d'invariants est bien «la stratégie fondamentale de la science dans l'analyse des phénomènes », comme le rappelle Jacques Monod (1970, p. 116). La réorientation ainsi proposée, il faut bien le dire, est en rupture totale avec les tendances dominantes. Quel que soit le domaine de spécialisation, les thèmes de recherche sont loin d'être définis à ce niveau d'abstraction et de généralité. Le discours sociologique en devient plus accessible au profane mais il est confronté à des réalités beaucoup plus fluctuantes dans le long terme et même dans le moyen terme. La prétention à faire œuvre de science relève alors très souvent de l'incantation. Il existe, certes, une tradition d'analyse formelle inspirée de l'œuvre de Simmel, que Raymond Boudon, il y a une vingtaine d'années, qualifiait d' 'important projet intellectuel, toujours bien vivant aujourd'hui» $(1984$, p. 48). Cette sociologie formelle est néanmoins restée en marge des courants majeurs de la sociologie et est largement méconnue à l'heure actuelle. On se propose d'explorer ici les perspectives qu'offre cette voie d'accès à la théorie générale ainsi que les problèmes qu'elle pose.

\section{LA FORME ET LE CONTENU}

Pour Simmel, on peut parler de faits sociaux - ou, si l'on préfère, de faits de société - lorsqu'il y a «action réciproque (Wechselwirkung) de plusieurs individus». Ces faits sociaux se présentent sous deux aspects que l'analyse doit séparer: leur forme et leur contenu. On peut discuter sur le choix de ces termes. Simmel lui-même n'y voit rien d'autre que des dénominations approximatives, permettant d'exprimer «l'opposition entre les éléments à distinguer». Bien sûr, dans tout phénomène social observable, ces deux éléments - forme et contenu sont indissolublement liés: ils «constituent une réalité concrète unitaire». Mais, paradoxalement, les modalités de l'action réciproque ne peuvent être «soumise à un point de vue scientifique unitaire» que si on «les détache des contenus» par la pensée. Pour que cette démarche analytique soit possible, il faut que la même forme apparaisse liée à «des contenus, des buts tout à fait différents» et que «le même intérêt de contenu» ait pour vecteur «des formes différentes». Dans la réalité, estime Simmel, il n'est pas rare que ces conditions soient effectivement remplies. Dans des groupes sociaux «dont les buts et les significations sont aussi différents qu'on peut l'imaginer», on observe des comportements formellement semblables. Ceci peut être aisément illustré par des exemples concrets: «domination et subordination, concurrence, imitation, division du travail, formation des partis, représentation, solidarité à l'intérieur en même temps que fermeture à l'extérieur: tout cela, et bien d'autres choses semblables, se retrouve dans la société étatique comme dans la communauté religieuse, dans la bande de conjurés comme dans le groupement d'intérêts économiques, dans l'école artistique comme dans la famille». Inversement, un contenu social tel que «l'intérêt économique»peut se présenter aussi bien «sous la forme de la concurrence que sous celle de l'organisation des producteurs » (Simmel, 1908, 1999, pp. 43-46). 
Afin de préciser sa pensée, Simmel use de la métaphore. La sociologie lui paraît entretenir avec les autres disciplines des sciences humaines «le même rapport que la géométrie avec les sciences physiques et chimiques »; elle s'intéresse à «la forme par laquelle la matière devient un corps empirique». On se rend compte en effet «que les mêmes formes géométriques se retrouvent sur les matières les plus diverses et que la même matière se présente dans les formes spatiales les plus diverses». Cette comparaison permet de mieux comprendre pourquoi forme et contenu, tout en étant analytiquement distincts sont liés empiriquement. Une forme spatiale «n'existe pour elle-même que dans l'abstraction »; elle ne peut avoir de réalité sensible «sans une matière dont elle est la forme». On songe à l'exemple devenu classique de la notion de triangle, distincte de tout objet triangulaire mais néanmoins indissociable de ses matérialisations empiriques. Il en va de même des formes sociales qui ne sont observables que dans leurs concrétisations en séquences d'interaction (ibid., pp. 49-50).

Comme toute sociologie, la sociologie formelle dont Simmel s'est fait l'avocat ne peut être mise en œuvre que sur un arrière-plan de psychologie explicitement formulée ou contenue implicitement dans les raisonnements. L'unité élémentaire de l'analyse est l'action réciproque ou l'interaction. Celle-ci exige que l'existence ou l'activité de l'un exerce une influence sur les états mentaux de l'autre. Dès lors, le contenu de l'interaction est tout ce qui lui confère un sens, tout ce qu'elle recèle, dans les termes de Simmel, comme "pulsions, intérêts, buts, tendances, états et mouvements psychologiques ». Il ne s'ensuit pas pour autant que l'interaction se ramène à la rencontre de psychologies individuelles. C'est précisément la combinaison contenu-forme qui en fait un objet d'analyse sociologique. La forme, c'est la manière dont s'opère la relation entre les individus impliqués dans l'interaction, compte non tenu de son objet (ibid., pp. 43-44). On peut y voir «les différents modèles », variés mais constants, selon lesquels se déroule la vie en société (Léger, 1989, p. 180). Pour Simmel, ces formes « conquièrent leur vie propre, une fonction libérée de tout enracinement dans un contenu» $(1917,1981$, p. 124).

\section{LA TRADITION DE SOCIOLOGIE FORMELLE}

La sociologie formelle a des antécédents philosophiques kantiens. Les formes de l'action réciproque telles que les conçoit Simmel sont des a priori sociologiques qui rendent la société possible, de même que les catégories a priori de l'entendement, selon Kant, rendent la connaissance possible. La filiation est très claire. Simmel se sépare néanmoins de Kant sur l'importance à accorder à la démarche cognitive du sujet. «Dans l'œuvre de Kant», écrit-il «l'activité mentale est dotée, à un degré jamais atteint jusque-là, de la capacité de mettre en forme les données de la représentation» $(1923,1984$, p. 62). Il s'ensuit que toute liaison entre les choses est pour Kant une production du sujet connaissant. Il n'en va pas de même du lien social, estime Simmel. Selon lui, ce lien s'accomplit «immédiatement dans les choses », en l'occurrence dans l'esprit des individus en interaction (1908, 1999, pp. 64-67). Pour Simmel, la Wechselwirkung, l'action réciproque, se substitue à l'activité cognitive du sujet individuel, ce qui constitue en fait une «sociologisation » de la pensée kantienne. 
Parmi les sociologues qui ont été influencés par Simmel, Célestin Bouglé et Eugène Dupréel sont ceux dont l'œuvre présente davantage qu'un intérêt historique et peut incontestablement rester une source d'inspiration pour les générations actuelles de chercheurs ${ }^{3}$. L'un et l'autre conservent la notion de base d'action réciproque - ou de relation réciproque - mais lui substituent le terme de « rapport». Pour Bouglé, il faut «remonter jusqu'à l'idée même de rapport, et tenir pour un fait social tout ce qui résulte des rapports quels qu'ils soient qui s'établissent entre les individus » (1896-1897, p. 152). Toutefois, certains rapports sociaux restent inchangés alors que les individus qu'ils unissent changent; on peut, «abstraction faite des différences propres aux individus, décrire, comparer, classer les rapports qui les relient: ce seront les formes sociales» (1907, p. 8). L'étude des formes se confond alors avec le point de vue sociologique lui-même: «se placer au point de vue géographique, c'est observer les formes terrestres, leurs conséquences et leurs causes. De même, se placer au point de vue sociologique, ce sera observer les formes sociales, leurs conséquences et leurs causes » (ibid., p. 31). En accord avec Bouglé tout comme avec Simmel, Dupréel voit dans le «rapport social» une catégorie formelle, indépendante du type d'actions ou d'états d'esprit qui y sont impliqués ${ }^{4}$. Dans sa Sociologie générale (1948), reprenant de façon plus lapidaire une définition déjà formulée en 1912 (p. 30), il pose qu'il existe un rapport social entre deux individus «lorsque l'existence ou l'activité de l'un influe sur les actes ou les états psychologiques de l'autre. Une influence réciproque, actuelle ou virtuelle [lui paraissant] le cas normal» (1948, p. 5). Rapports sociaux d'imitation (Tarde), rapports sociaux modelés par la pression de la collectivité (Durkheim), rapports sociaux définis par la convergence des états d'esprit (Giddings) sont des catégories particulières de rapports sociaux; l'essentiel réside dans la notion même de rapport (Dupréel, 1912, pp. 35-40). L'influence qui s'exerce dans le rapport social peut se manifester de trois façons: par la force ou la contrainte, par la persuasion au sens le plus large ou encore par l'échange d'avantages. Ces trois modes d'influence - qui sont également des catégories formelles - seront à la base d'une typologie des groupes sociaux qui peut servir de base à des propositions théoriques formelles ou substantielles, selon qu'on s'en tient à la logique d'enchaînement des rapports élémentaires ou qu'on y introduit des contenus spécifiques. Mais, qu'on s'inscrive dans une logique de contrainte, de persuasion ou d'échange, il est hors de doute pour Dupréel qu'il existe «des lois formelles en dehors desquelles aucune vie sociale n'est possible» (Dupréel, 1949, pp. 154-155). Ces «lois formelles» sont en fait des principes de compatibilité ou d'incompatibilité. Une forme d'influence ayant été mise en œuvre, elle s'avère compatible ou incompatible avec d'autres modalités d'action.

C'est chez Dupréel qu'apparaît le plus clairement - de façon quasi didactique - le lien entre l'action réciproque et les catégories formelles. L'action réciproque élémentaire - à laquelle s'identifie le rapport -, se définit comme un processus à

3 Ludwig von Wiese, souvent considéré comme le continuateur de Simmel, a renoncé à la distinction entre forme et contenu pour ne garder que l'élément relationnel de la sociologie simmelienne (Von Wiese, 1928).

4 Concernant la sociologie de Dupréel et ses fondements philosophiques, voir mon article Eugène Dupréel, philosophe, sociologue et moraliste (Coenen-Huther, 2006, pp. 97-118). 
caractère très général qui s'actualise dans des formes diverses. Mais les formes sociales n'existent que par l'interaction et les états mentaux qu'elle suscite. Elles exigent des individus conscients pour lesquels une séquence comportementale puisse revêtir un sens. C'est le sens attribué par les acteurs parties prenantes à l'interaction qui permet de parler de contrainte, de persuasion ou d'échange. C'est aussi cette attribution de sens qui permet d'introduire dans le rapport la «double contingence» signalée ultérieurement par Parsons et par Luhmann. A ce stade, on peut vouloir sortir de l'analyse purement formelle et réintroduire des contenus. Cependant, le cheminement intellectuel qui conduit à un cas de figure réunissant forme et contenu passe par davantage de précision dans l'analyse formelle. La contrainte présente dans un rapport ou dans un groupe - c'est-à-dire un rapport de rapports - peut être morale ou matérielle, insidieuse ou brutale. La nature de la contrainte ayant ainsi été graduellement précisée, son contenu social peut faire l'objet d'une comparaison mettant en évidence les similitudes et les différences avec d'autres cas relevant de la contrainte. On retrouve ainsi l'alternance entre théories formelles et substantielles à laquelle conduit la stratégie de comparaison constante préconisée par Glaser et Strauss.

La sociologie formelle, il faut le préciser, n'est pas une microsociologie. Pas plus chez Dupréel que chez Simmel ou chez Bouglé, elle ne se limite aux formes élémentaires d'interaction. Il s'agit d'une perspective sociologique pour laquelle l'ordre social est constitué de rapports qui s'interpénètrent et comportent des termes communs. L'imbrication des rapports sociaux complémentaires «se prolonge en des chaînes de longueur indéfinie et leurs implications terme à terme se multiplient en réseaux inextricables » (Dupréel, 1948, pp. 18-19). La complémentarité dans la durée suscite une structuration dont découle la formation des classes sociales et des hiérarchies à l'échelle des sociétés globales. Le potentiel macrosociologique de l'analyse des formes a été mis en évidence par Bouglé dans son étude sur les «idées égalitaires ». Le raisonnement s'y fonde sur la notion de compatibilité: "pour qu'une idée pénètre une société, il faut qu'il y ait, entre la nature de celle-là et la structure de celle-ci, une sorte d'harmonie préétablie» (1899, p. 84). Si une telle harmonie existe, c'est au niveau des formes qu'il faut la découvrir. Selon Bouglé, c'est «à l'intérieur de cette civilisation qu'on appelle occidentale» que l'idée d'égalité est apparue (1899, p. 64). Ce jugement ne comporte aucun essentialisme: ce sont «les formes sociales propres à la civilisation occidentale [qui] sont aussi les plus favorables au succès des idées égalitaires» (ibid., p. 236). Les facteurs qui entrent en jeu dans l'analyse sont des processus formels: localisation vs délocalisation, centralisation vs décentralisation, mode vs coutume, législation universaliste vs particulariste, etc. Soucieux de dépasser le discours programmatique et d'appliquer l'analyse formelle à des objets concrets, Bouglé met en garde contre la «fièvre classificatrice» qui s'emparera ultérieurement de Ludwig von Wiese: «une science», note-t-il «ne saurait se contenter de classer des formes: elle veut découvrir, entre certains phénomènes donnés, certaines relations constantes, et prouver que les uns varient en fonction des autres. C'est ce que la sociologie pourra tenter d'établir en observant les conséquences des formes qu'elle aura classées » (ibid., p 10).

Le désir d'utiliser la notion de forme sociale pour mettre en évidence des constantes de la vie en société - par opposition à la diversité et à la variabilité des contenus - a conduit certains commentateurs a établir un parallèle entre la socio- 
logie formelle et le structuralisme. François Léger voit dans la sociologie formelle «une théorie dont il est permis de dire ... qu'elle s'apparente au structuralisme dans la mesure où elle assigne comme tâche à la sociologie de décrire des configurations abstraites » $(1989$, p. 191). On ne décèle aucune filiation directe entre la pensée de Simmel et celle de Lévi-Strauss mais le rapprochement n'en est pas moins intéressant, encore qu'il soit inexact que l'objectif de la sociologie formelle soit de décrire «des configurations abstraites ». L'ambition scientifique est certainement semblable dans les deux cas. Selon Lévi-Strauss, «à partir du moment où de nombreuses formes de la vie sociale - économiques, linguistiques, etc - se présentent comme des rapports, la voie s'ouvre à une anthropologie conçue comme une théorie générale des rapports » (1958, p. 110). Opposant formalisme et structuralisme, Lévi-Strauss écrit néanmoins que «la forme se définit par opposition à une matière qui lui est étrangère; mais la structure n'a pas de contenu distinct: elle est le contenu même, appréhendé dans une organisation logique conçue comme une propriété du réel» (1973, p. 139). En dépit de la distinction proposée entre forme et structure - distinction dont la pertinence est liée à une acception des termes de «forme» et de «structure» qui n'est pas unanimement acceptée -, l'idée ainsi énoncée rejoint la position théorique de Simmel pour qui la distinction analytique entre forme et contenu recouvre, on l'a vu, «une réalité concrète unitaire». Le projet scientifique de la sociologie formelle est également d'appréhender la «matière sociale» dans «une organisation logique» (Léger, 1989, p. 345). Mais Lévi-Strauss s'interroge sur la nature des constantes, ou invariants, de la vie en société. Et c'est une question qu'on se doit d'examiner ici par le truchement d'une réflexion sur la nature des formes de la vie sociale.

\section{LA NATURE DES FORMES SOCIALES}

L'existence de conditions formelles de la vie en société étant donnée, la question est de savoir de quelle nature sont ces formes et à quel niveau elles opèrent. Formuler ce genre d'interrogation, c'est inévitablement retrouver le problème classique de la nature de la relation entre le sujet connaissant et l'objet de sa connaissance. Dans une optique piagétienne, la connaissance que nous pouvons acquérir des formes sociales résulte d'une relation entre trois éléments: un sujet, un objet et une structure opératoire (Piaget, 1967, pp. 3-4). Il est possible de définir et de localiser les formes de la vie en société à partir de chacun de ces éléments.

Pour Dupréel, «le rapport social est une forme qui devient une réalité lorsqu'on trouve cette forme ordonnant les démarches des êtres vivants. Cette forme impose à cette réalité ses propriétés (...) Il est permis de parler de conditions $a$ priori de la vie en société, indépendamment de l'espèce des individus et de leurs particularités, on peut faire la sociologie de Sirius sans tomber dans la fantaisie » (1948, p.9). Selon cette conception, les formes sociales ne concernent pas uniquement les sociétés humaines. Il y a des lois de la vie en commun qui ne sont liées ni à la volonté des hommes ni même à la nature humaine; elles «règleront au même titre le comportement des sociétés animales que les affaires des sociétés où l'on parle et où l'on pense» (ibid., p. 51). Dans la perspective évolutionniste qui est celle de Dupréel, l'homme fut un être social avant d'être humain et les formes 
sociales se sont imposées à lui à un stade pré-humain. Ceci concorde avec les avancées récentes en éthologie qui nous font découvrir des formes sociales élémentaires chez les grands singes anthropoïdes. L'idée en résulte que ces formes se retrouvent dans la vie de toute espèce animale grégaire. Les éléments formels de la vie en société participeraient alors du phénomène de la vie et échapperaient ainsi à la causalité classique (Hersch, 1993,p. 247).

On peut au contraire privilégier une hypothèse kantienne modifiée et estimer que les formes sociales sont liées à un objet défini de façon plus précise comme les comportements d'une espèce particulière, à savoir l'espèce humaine et ses caractéristiques propres. Cette manière de voir est plus conforme à la vision simmelienne d'éléments analytiquement distincts mais nécessairement liés. De ce point de vue, la sociologie formelle de Simmel a pu être présentée comme «une synthèse originale de néokantisme (l'opposition des formes et des contenus) et de vitalisme (l'interaction)» (Vandenberghe, 2001, pp. 42-43). Le rapport social, l'action réciproque, est un rapport dont le contenu est nécessairement humain car il implique les perceptions d'individus humains avec ce que cela implique d'imprévisibilité. On se rappellera à cet égard la célèbre formule marxienne: «L'animal n'est en rapport avec rien, ne connaît somme toute aucun rapport. Pour l'animal, ses rapports avec les autres n'existent pas en tant que rapports. La conscience est donc d'emblée un produit social» (Marx et Engels, 1846, 1968, p. 59).

Il est possible d'aller plus loin dans la spécification et de considérer les formes sociales non seulement comme des conditions qui s'imposent en quelque sorte «de l'extérieur» à notre espèce par des effets de structuration, mais comme une construction propre à l'esprit humain. Cette hypothèse implique une certaine conception de la nature humaine qui s'oppose au «tout-sociologique ${ }^{5}$ souvent affirmé de manière dogmatique dans les milieux de sociologues. La thèse des formes sociales-productions de l'esprit humain est celle de Claude Lévi-Strauss et c'est en cela que son œuvre revêt une pertinence directe pour notre objet. Pour Lévi-Strauss, il y a une «activité inconsciente de l'esprit» qui consiste à «imposer des formes à un contenu ». Dans la mesure où ces formes restent «fondamentalement les mêmes » dans le temps et dans l'espace, il doit y avoir des schèmes très généraux, n'existant pas en nombre illimité et se ramenant à des «relations de corrélation et d'opposition» dont les individus engagés dans la vie sociale «ne sont pas conscients » (Lévi-Strauss, 1983, pp. 28-31). On est confronté à ce sujet à deux hypothèses extrêmes: «celle d'un esprit plastique, passivement modelé par des influences extérieures, et celle de lois psychologiques universelles parce que innées ». A l'heure actuelle, il semble raisonnable de chercher une voie moyenne entre ces «deux déterminismes »: l'action du milieu et l'équipement mental de l'homme. Lévi-Strauss nous y invite en cherchant l'articulation de ces «deux ordres de détermination» dans notre «activité cérébrale», permettant la connaissance par le «codage» de données «enregistrées par les sens selon des traits distinctifs » (1983, pp. 146 et 165). Ce qui incite Lévi-Strauss à rechercher des mécanismes universels de la pensée humaine, c'est son intérêt pour la thèse de

5 J'emprunte cette expression au biographe de Claude Lévi-Strauss, Denis Bertholet (2003, p. 333). 
Chomsky selon laquelle il y aurait, sous la multiplicité des langues humaines, une «structure profonde» ou «forme» qui serait la même. Cette forme innée, définissant une sorte de «langue mère », serait caractéristique de l'espèce humaine. Dans cette perspective, toute forme d'apprentissage soumise aux influences de l'environnement constitue une sélection parmi des potentialités qui, pour n'être pas illimitées n'en sont pas moins beaucoup plus nombreuses que leurs actualisations dans une culture particulière. On aurait ainsi des formes attribuables à des caractéristiques de l'espèce humaine, s'imposant à des contenus, tributaires d'environnements culturels divers.

\section{L'HÉTÉROGÉNÉITÉ DES FORMES SOCIALES}

Les divers points de vue relatifs à la nature et à l'origine des formes sociales portent généralement sur la notion de forme sociale prise dans sa généralité. Hors, les formes qui sont énumérées à titre d'exemples par les auteurs acquis à l'idée d'une opposition analytiquement féconde entre forme et contenu, sont loin de constituer une catégorie homogène. Au-delà des approximations reconnues par Simmel, il est possible de distinguer parmi les exemples cités et commentés, des catégories différentes de formes auxquelles peuvent s'appliquer des diagnostics sociologiques différents.

1) Il y a tout d'abord ce qu'on pourrait appeler des formes pures, qui sont les plus proches des formes géométriques ayant servi de référence métaphorique à Simmel. C'est à l'existence de telles formes que Bouglé nous rend attentifs lorsqu'il se demande si une société est «grande ou petite, durable ou momentanée, homogène ou hétérogène, totale ou partielle, organisée ou inorganisée, hiérarchique ou égalitaire, etc » $(1907$, p. 10). Ce sont de telles formes qui ont le caractère de généralité le plus incontestable et qui ont incité Dupréel à déclarer qu'elles pourraient aussi bien jouer un rôle dans la «sociologie de Sirius» (1948, p. 9). Il s'agit certainement des formes qui se prêtent le plus aisément à l'examen de leur influence par l'analyse comparative. La taille d'un groupement social, sa durée, sa plus ou moins grande homogénéité, etc, ont des conséquences globalement prévisibles même s'il ne s'agit que de tendances. Ces formes pures pèsent de toute évidence sur les contenus sociaux particuliers.

2) Il existe ensuite des formes localisées, produits de l'histoire ou d'une culture particulière. Ainsi en est-il des formes sociales que Bouglé associe à l'émergence des idées égalitaires. Le jeu de ces formes n'a de sens que par rapport au déroulement de l'histoire de la civilisation occidentale. S'il est possible d'associer, dans ce contexte particulier, égalitarisme, centralisation et unification du territoire, c'est en référence à «l'éclipse de l'égalité» contemporaine de la féodalité, ce «mouvement de localisation» anarchique qui avait «pour longtemps morcelé les intérêts et dispersé les pouvoirs» (Bouglé, 1899, p. 211). Dans ce cas, ce sont les contenus sociaux qui délimitent le cadre dans lequel les formes acquièrent une valeur analytique. La fonction qu'on peut attribuer aux éléments formels est ici moins d'assurer une certaine prévisibilité que de rendre possible une interprétation de faits déjà acquis. 
3) Une troisième catégorie de formes peuvent être qualifiées de formes idéaltypiques dans la mesure où elles n'ont pas trait à une réalité empiriquement observable mais bien à une réalité stylisée. On en trouve un bon exemple chez Dupréel. Celui-ci estime que «tous les associés, de quelque nature qu'ils soient, doivent se faire un minimum de concessions pour se rendre compatibles les uns avec les autres» et il y voit une «loi de compatibilité » se trouvant au nombre de ces « lois formelles en dehors desquelles aucune vie sociale n'est possible» $(1949$, p. 155). En fait, cette règle de compatibilité n'est indispensable qu'à une vie sociale sans conflit, ou marquée seulement par des tensions mineures. Dans la vie réelle, il ne manque pas d'exemples de processus sociaux dépourvus d'un minimum de compatibilité entre les parties en présence. Ce principe de compatibilité, tel qu'il est présenté par Dupréel, autorise la comparaison entre les formes sociales inspirées de l'œuvre de Simmel et les types idéaux weberiens. Les premières comme les seconds ont valeur de modèles ${ }^{6}$ et peuvent ne pas se trouver «à l'état pur dans la réalité » (Vandenberghe, 2001, p. 40).

4) Une autre catégorie est constituée de formes en acte. On entend par là des processus sociaux, parfois complexes, dont le déroulement ne s'explique que par l'existence de formes sous-jacentes qui les structurent. L'élément formel de «dimension» en fournit une illustration. Selon Simmel, «un groupe, à partir d'une certaine dimension, doit élaborer, pour se maintenir et se développer, des règles, des formes et des organes dont il n'a pas besoin auparavant (...) d'un autre côté, des cercles plus restreints présentent des qualités et des actions réciproques qu'ils perdent inéluctablement dès que le nombre de leurs membres s'étend». Simmel fait observer à ce sujet que la dimension ne se présente pas seulement sous la forme d'une grandeur relative mais aussi sous la forme d'une grandeur numérique absolue (Simmel, 1908, 1999, p. 81). La tendance très générale à la constitution d'oligarchies dirigeantes, dont Roberto Michels a cru pouvoir faire une «loi d'airain», est la concrétisation de telles formes en acte (Coenen-Huther, 2004, pp. 38-43).

5) Il est enfin possible de distinguer parmi les formes mentionnées dans la littérature, des formes au second degré. Il s'agit de configurations sociales qui présentent certes un caractère formel mais qui peuvent être ramenées à des formes plus générales et plus simples. Schmoller, cité par Bouglé, présente la tendance à constituer un droit de plus en plus égalitaire comme la conséquence «de la révolution qui, au XIIIe siècle, unit les hommes dans les villes, et fit, d'un peuple de paysans, un peuple de citoyens ». D'importantes transformations du droit sont ainsi mises en rapport avec le milieu urbain. Il s'agit là, comme le fait observer Bouglé, de l'action d' « une synthèse de formes sociales élémentaires» (Bouglé, 1907, p. 16). Il en va de même lorsque Tarde met en relation le passage des sociétés de type aristocratique aux sociétés de type démocratique avec la substitution de la mode à la coutume $(1895,1904, \mathrm{pp}$. 265-394). Dans les deux cas, nous avons affaire à des complexes de formes

6 Voir à ce sujet mon article Le type idéal comme instrument de la recherche sociologique (Coenen-Huther, 2003, pp.531-547). 
élémentaires - imitation et distinction - dont seuls les cadres de référence sont différents. La généralité du mécanisme n'apparaît qu'à la suite de la décomposition de formes au second degré en formes plus générales.

Cet essai de classification des formes sociales apparaissant dans les travaux de quelques classiques n'est qu'une ébauche. Une typologie plus élaborée devrait permettre de surmonter ce qui peut apparaître de prime abord comme des contradictions internes de la sociologie des formes.

\section{LES FORMES SOCIALES ET LA PSYCHOLOGIE}

Le sentiment d'avoir affaire à un réductionnisme psychologique est certainement ce qui a détourné le plus souvent les sociologues de la sociologie formelle. Dans une recension critique de la Sociologie générale d'Eugène Dupréel, le Doyen Davy note qu' «il ne nous paraît pas possible de fonder, comme M. Dupréel semble trop enclin à le faire, la sociologie sur une science abstraite des rapports sociaux, science qui ramène fatalement à la psychologie individuelle» (Davy, 1948-49, p. 123). Auparavant, Raymond Aron, commentant les travaux de Simmel, avait écrit que «ces formes sociales créées par l'action réciproque (Wechselwirkung) ... ne s'expliquent en dernière analyse que par la psychologie» $(1935,1981$, p. 6). Il est vrai que toute sociologie se fonde, consciemment ou inconsciemment, sur une psychologie de convention. Raymond Boudon a fait observer à cet égard que le sociologue a toujours à l'esprit un «acteur banalisée (1986, p. 22) et que Durkheim lui-même appuyait ses raisonnements sur une psychologie implicite (1994, pp. 99-122). Cependant, la sociologie formelle est une sociologie relationnelle et son unité élémentaire n'est pas l'individu mais l'interaction. Rien dans les travaux de Simmel ou de ceux qui s'en inspirent ne suggère la réduction des faits sociaux à des actions individuelles (Vandenberghe, 2001 , p. 49). Chaque situation d'interaction - chaque rapport social - comporte un surplus collectif ${ }^{7}$ constitué par le «bagage social et culturel» que chaque individu y apporte. Les actions réciproques s'effectuent «selon des formes typiques» affectées par l'environnement social. Au surplus, l'imbrication des actions réciproques - ce que Dupréel appelle la complémentarité des rapports sociaux suscite des effets émergents qui sont à la base des structures sociales. Ces effets émergents ne se réduisent donc pas à l'agrégation de comportements individuels. On comprend dès lors que, parmi les sociologues modernes, ce sont les sociologues d'orientation interactionniste qui se sentent le plus en affinité avec la tradition de recherche liée à la sociologie formelle. Glaser et Strauss, avec leurs «contextes de conscience», en constituent un bon exemple. Les situations d'interaction peuvent être associées à l'activité inconsciente de l'esprit humain, comme le fait Lévi-Strauss, mais pour ce dernier, «l'inconscient cesse d'être le refuge des particularités individuelles, le dépositaire d'une histoire unique (...) Il se réduit à (...) la fonction symbolique, spécifiquement humaine, sans doute, mais

7 Ce qui faisait dire à Auguste Comte que d'innombrables individus, vivants et morts, sont présents dans chaque relation sociale élémentaire. 
qui, chez tous les hommes, s'exerce selon les mêmes lois» (1950, p. XXXI). Ce que l'analyse des formes sociales remet sérieusement en question, ce n'est pas l'autonomie de la sociologie face à la psychologie mais bien un certain sociologisme prenant position de manière dogmatique dans le débat entre l'inné et l'acquis.

\section{LES FORMES SOCIALES ET LA RECHERCHE EMPIRIQUE}

Au cours de mes propres travaux, j'ai eu fréquemment l'occasion de prendre appui sur les éléments formels des situations observées et de chercher à élargir la portée d'une observation par le recours à la distinction entre forme et contenu, dans l'esprit de Simmel. Donnons-en ici quelques exemples, extraits de mon livre Observation participante et théorie sociologique (1995) auquel je renvoie le lecteur pour davantage de détails sur les thèmes abordés.

\section{Les enfants du kibboutz}

Un kibboutz est une communauté de vie et de travail. Le jeune, né dans un kibboutz et élevé en son sein, est à la fois un enfant de la communauté et un membre potentiel du collectif de travail. Ce double statut est la source d'une incertitude normative qui se manifeste particulièrement lorsqu'il arrive à maturité. Pendant toute son enfance, le kibboutz est pour lui une grande famille et l'univers particulariste des rapports parents-enfants colore d'affectivité ses relations avec ses aînés. Lorsqu'il arrive au seuil de l'âge adulte, les normes universalistes de la vie de travail deviennent pour lui d'application et entrent en conflit avec les normes particularistes de son enfance (1994, chap. I). Cette tension entre deux systèmes de normes est la conséquence directe d'un double statut, dans un groupe de dimension restreinte où les effets de cette dualité ne peuvent être neutralisés par une segmentation de l'environnement social. Un raisonnement centré sur les éléments formels de la situation nous éloigne du kibboutz et de son contenu social spécifique, pour nous amener à conclure qu'une dualité de statut, dans un groupement ayant les caractéristiques d'un «système total», met en présence deux systèmes de rôles aux exigences contradictoires et constitue une source interne de tensions. Nous avons affaire ici à un jeu de formes pures qui comporte un potentiel de prévisibilité incontestable.

\section{Les relations à plaisanteries}

Les ethnologues ont eu leur attention attirée par un type de relations assez particulier qu'on est convenu d'appeler «parenté à plaisanteries ». Il s'agit d'une relation ritualisée entre deux personnes unies par un lien de parenté - par exemple l'oncle maternel et le neveu utérin - dans laquelle l'une taquine l'autre ou s'en moque alors que l'autre n'est pas censée en prendre ombrage (1995, chap. III). Dans ce type de relations, la moquerie est toujours une façon de combiner la sympathie et l'antagonisme. C'est en raison de cette ambivalence que la mesure d'antagonisme incluse dans la relation s'exprime sous la forme mitigée de la plaisanterie. L'ambivalence de la relation est le reflet de l'ambiguité d'une situation 
où se combinent deux loyautés: celle qui est ressentie à l'égard du groupe d'origine et celle que l'on éprouve à l'égard du groupe avec lequel s'établit une certaine forme d'alliance. La plaisanterie correspond donc à la volonté de neutraliser une source potentielle de tensions. Dans les sociétés archaïques, les relations interpersonnelles sont modelées principalement en fonction de l'appartenance des individus à des collectivités. Dans nos sociétés occidentales développées, aucune norme contraignante ne nous impose de traiter un individu en fonction de l'attitude adoptée à l'égard de toute une parentèle; une source de tensions possibles disparaît donc. Il n'en existe pas moins des relations porteuses de tensions latentes et des comportements permettant à la fois d'exprimer ces tensions et d'en limiter les effets. Et la plaisanterie y est aussi, dans certaines circonstances, la marque de l'ambivalence. Ce qui est différent, ce sont les sources de tensions et d'ambivalence. La recherche empirique a mis en évidence des relations d'affinité qui pourraient être menacées par des éléments perturbateurs tels qu'un antagonisme de valeurs, un écart de statuts, une ambiguïté de rôles ou un conflit de normes. Ces éléments peuvent être neutralisés par la plaisanterie et l'humour. Voilà donc des situations d'interaction qui appartiennent à des mondes sociaux différents et qu'apparemment tout sépare. Si l'on s'en tient à la forme des relations, on peut néanmoins y déceler un même mécanisme de réduction de tensions dans des contextes différents. Ici aussi, des formes pures exprimant une combinaison d'identité et d'altérité sont en jeu. Sous-jacents à ces formes, il pourrait y avoir des mécanismes généraux de l'esprit humain, tels que ceux dont Lévi-Strauss envisage l'existence.

\section{Le milieu hospitalier}

Le milieu hospitalier est le cadre institutionnel de relations interpersonnelles diverses. Il faut distinguer à cet égard plusieurs systèmes d'interaction différents dans le même cadre organisationnel. Un premier système régit les relations entre patients et membres du personnel, un deuxième régit les relations entre patients, un troisième régit les relations entre membres du personnel.. Chacun d'eux est constitué d'un ensemble de rôles et d'attentes de rôles répondant à des exigences différentes, parfois contradictoires (1995, chap. V). Quand il s'agit d'un hôpital universitaire, ces trois systèmes d'interaction se trouvent compliqués par le fait qu'il ne s'agit pas seulement d'un organisme de soins mais aussi d'un élément d'une faculté de médecine où l'on fait de la formation et de la recherche. Le personnel se trouve engagé quotidiennement dans deux systèmes d'interaction différents: avec les patients d'une part, avec les autres membres d'un personnel diversifié et hiérarchisé d'autre part. Il s'ensuit que certains rituels hospitaliers n'ont pas le patient pour objet premier. Des comportements peuvent être fonctionnels dans le cadre d'un système d'interaction tout en étant dysfonctionnels dans le cadre d'un autre. C'est le cas, par exemple, des jeux de rôles accompagnant la visite matinale du «Patron»: fonctionnels du point de vue des carrières médicales et académiques, ils sont dysfonctionnels, ou tout au moins dénués de fonction, dans le cadre du système patients-personnel. Si nous nous écartons en pensée de l'hôpital et de ses spécificités, nous nous trouvons face à une organisation complexe au sein de laquelle les diverses catégories d'acteurs mettent en œuvre des échelles de préoccupations et de prestige différents. Le caractère 
plurifonctionnel de l'institution hospitalière suffit à rendre compte de certaines tensions latentes. Bien sûr, le contenu social - la maladie, la souffrance, les soins à donner - ne doit pas être perdu de vue mais des formes en acte assurent une dynamique organisationnelle qui peut en être analytiquement détachée.

\section{Le monde de l'alpinisme}

Le Club Alpin de Genève organise régulièrement pour ses membres des courses collectives en haute montagne (1995, chap. II). Dans le «contexte exposé » d'une ascension ou d'une traversée de glacier s'instaure une hiérarchie informelle basée sur le niveau de performance de chaque alpiniste. Mais quand un groupe du Club Alpin s'installe dans un refuge de haute montagne pour y passer la nuit, le contexte exposé de la lutte avec la montagne fait place au «contexte protégé» de la cabane. Un clivage apparaît assez vite entre une petite clique de responsables au verbe haut et la piétaille réduite au rang de public. L'échelle de statuts et les règles de sociabilité changent dès que le temps de l'effort et du danger est passé. Ce qui compte alors pour assurer le prestige, c'est l'ancienneté dans l'organisation (les «années de sociétariat»), ce sont les bouteilles de rouge vidées en commun. D'autres talents que ceux du montagnard aguerri sont à l'honneur et ce sont des talents sociaux: aptitude à raconter, à blaguer, à jouer aux cartes, à trinquer... Le « chef de course » et son entourage le plus direct accueillent avec bienveillance les entorses les plus évidentes à l'ascèse des sportifs : pratiques alimentaires discutables (la fondue à la veille d'une ascension), culte du vin, cabanes transformées en tabagies, etc. Il est possible de mettre en évidence deux modèles de participation associative qui sont sous-jacents aux deux contextes d'interaction antithétiques. Le premier est de type «médiation »: l'association est l'instrument de la pratique d'un sport. Le second est de type «allégeance»: l'association rend possible les retrouvailles régulières entre copains. D'un point de vue formel, les deux modèles renvoient à l'opposition entre Gesellschaft et Gemeinschaft ou encore à la distinction entre urban- et folk-societies, ce qui autorise des généralisations allant bien au-delà du monde de l'alpinisme. Quant à la modification des critères d'excellence d'un contexte à l'autre, elle se rattache formellement à des processus d'institutionnalisation avec déplacement des buts et à des processus d'oligarchisation. L'habitude d'opérer la distinction entre forme et contenu et d'appliquer cette distinction aux domaines les plus divers permet le passage des interactions entre alpinistes aux travaux de Pareto et de Michels sur les élites, et vice-versa. Les formes sociales repérées dans ce cas-ci peuvent être qualifiées de formes localisées en ce sens que leur fécondité analytique se limite à l'univers social où l'opposition de ces formes de sociabilité a un sens. Il s'agit d'une localisation dans le temps et dans l'espace. Leur potentiel de généralisation n'en reste pas moins élevé.

\section{Les files d'attente en Russie soviétique}

La file d'attente faisait partie intégrante du système de distribution de type soviétique dont elle symbolisait l'inefficacité et l'incapacité à s'adapter aux besoins des consommateurs (1995, chap. VI). En Russie soviétique, la file d'attente n'était pas un simple système d'interdépendance, à savoir une situation d'interaction sans relations de rôles. Chaque membre de la file faisait l'objet 
d'attentes de rôle spécifiques. Il y avait, par exemple, le rôle de «la personne qui précède», autorisée à s'absenter, et celui de «la personne qui suit», appelée à confirmer le droit de réintégrer la file. Dans ce contexte de pénurie et de lourdeur bureaucratique, la file d'attente tendait à l'auto-organisation et ce collectif émergent était extrêmement méfiant à l'égard des interventions régulatrices extérieures. Dès qu'on s'écartait du cas banal de la file de durée brève ou moyenne, l'auto-organisation allait bien au-delà des règles gouvernant les conduites en situation d'interaction immédiate. Lorsque l'attente durait plusieurs jours, voire plusieurs semaines ou plusieurs mois, il y avait choix - parfois élection - d'un leader, constitution d'une liste écrite, ébauche de stratégies collectives. Ces formes d'auto-organisation parfois très élaborées étaient nées des carences du système de distribution, de l'apparente indifférence des autorités et de la méfiance à l'égard du personnel fonctionnarisé du secteur des services; une population livrée à elle-même adoptait des stratégies de survie souvent ingénieuses. Tout ceci n'aurait plus qu'un intérêt historique si l'on n'extrayait pas les éléments formels de la situation. De manière beaucoup plus générale, le sentiment d'aliénation, le sentiment de n'avoir rien à attendre des détenteurs de positions de pouvoir, suscite des phénomènes d'organisation informelle. C'est ce qu'indique de l'une ou l'autre façon l'expérience des institutions totales, du monde carcéral ou de l'univers concentrationnaire. Selon la logique appliquée autrefois par Merton aux activités de la «machine politique» américaine, l'émergence du collectif de la file d'attente soviétique peut être interprétée comme un mécanisme de substitution. Et de tels mécanismes de substitution peuvent être liés tant au racket organisé qu'à des activités d'entraide. De l'auto-organisation protectrice à la «protection» imposée, il n'y a souvent qu'un pas. Les formes sociales séparables analytiquement de leur contenu historique particulier sont des formes en acte: ce sont des mécanismes informels d'auto-organisation en l'absence de régulation surplombante. Leur pouvoir de prédiction est limité mais bien réel: on peut s'attendre à ce que des situations structurellement semblables provoquent des réactions du même type, avec des effets pervers comparables.

Les cinq exemples qui viennent d'être brièvement évoqués relèvent de domaines d'investigation très variés et de spécialisations très différentes de la sociologie. L'analyse formelle les met au service de la théorie générale et celle-ci leur confère un surplus de valeur par rapport à l'approche monographique. Les avancées théoriques qu'on peut espérer obtenir par ces efforts de généralisation et d'abstraction se distinguent par un ancrage dans la réalité empirique.

\section{POUR CONCLURE}

La sociologie formelle a pour objet les formes qui structurent les processus d'interaction. En adoptant cet angle d'analyse, c'est le social lui-même qu'elle explore. Elle est inséparable, on l'a vu, d'une conception relationnelle de la sociologie. Dans cette perspective, l'action réciproque est le mécanisme essentiel de la vie en société: elle offre une voie moyenne entre le holisme et l'atomisme social. Dès lors, l'ordre social est constitué de rapports dont les individus sont les termes et qui s'agencent en configurations de rapports. Leur imbrication suscite des phénomènes d'émergence qui ont des effets structurants. Il ne s'agit cependant 
pas d'agrégation de comportements individuels mais bien d'agrégation de situations d'interactions modelées par l'environnement social. Méthodologiquement, la sociologie formelle est un interactionnisme. L'action réciproque n'est pas un phénomène de nature psychologique bien qu'il ait des retombées d'ordre psychique. C'est le fait de la vie en commun, le fait de la vie en société, qui pèse sur les consciences individuelles.

La condition de la mise en œuvre du programme de la sociologie formelle est le maintien de la stabilité d'un appareil conceptuel qui a fait ses preuves. Pour que les procédés de raisonnement hérités de la tradition d'analyse formelle puissent être fructueux, il est indispensable de se situer dans une optique de constitution d'un savoir cumulatif, les études monographiques d'aujourd'hui devenant les matériaux de la théorie générale de demain. Plus que toute autre orientation théorique, la sociologie formelle est liée au souci de cumulativité en sociologie et plus que toute autre orientation, elle peut contribuer à cette cumulativité. L'abandon régulier de concepts qui ont fait la preuve de leur utilité, les innovations de vocabulaire qui n'ont d'autre fonction que de se donner à bon compte un air d'originalité ne sont évidemment pas favorables à la perspective ainsi tracée (CoenenHuther, 2005a, 2005b).

Les antécédents philosophiques kantiens de la tradition d'analyse formelle sont incontestables. Bien au-delà de cette filiation kantienne, on pourrait remonter à l'ontologie aristotélicienne: la matière est l'être en puissance, en état d'indétermination; la forme est l'être en acte, ce qui confère la détermination à la matière. On a ici en germe l'idée simmelienne de la forme et du contenu, indissociables bien qu'analytiquement séparables. En dépit de son enracinement philosophique - ou peut être grâce à lui - cette façon de concevoir l'élaboration théorique en sociologie est éminemment favorable à la fécondation réciproque de la théorie générale et de la recherche empirique. On se doit d'ailleurs de faire observer ici que l'empirisme a montré ses limites au cours des dernières décennies. Au contraire, des variantes d'apriorisme kantien prolongées par le constructivisme piagétien ont conservé leur capacité à fournir des hypothèses fécondes à la recherche. Cette orientation qui est inséparable d'une certaine conception de la nature humaine implique que le social ne se réduit pas à l'articulation de stratégies conscientes. La structuration par émergence obéit à une logique qui n'est pas - ou pas nécessairement - celle des acteurs en présence.

\section{Université de Genève}

\section{RÉFÉRENCES BIBLIOGRAPHIQUES}

Aron, Raymond, La sociologie allemande contemporaine, Paris, PUF, 1935; Quadrige, 1981.

Becker, Howard, Outsiders. Studies in the Sociology of Deviance, New York, MacMillan, 1963.

Bertholet, Denis, Lévi-Strauss, Paris, Plon, 2003.

Boudon, Raymond, L'idéologie. L'origine des idées reçues, Paris, Fayard, 1986.

Boudon, Raymond, «Introduction » in: Simmel, Georg, Les problèmes de la philosophie de l'histoire, Paris, PUF, Coll. «Sociologies », 1984.

Boudon, Raymond, «Durkheim et Weber: convergences de méthode», in: Monique Hirschhorn et Jacques Coenen-Huther, Eds, Durkheim et Weber, Vers la fin des malentendus? Paris, L'Harmattan, 1994, pp. 99-122. 
Bouglé, Célestin, «Notice sur The Principles of Sociology par F. H. Giddings », L'Année sociologique, Vol. I, 1896-1897.

Bouglé, Célestin, Les idées égalitaires. Etude sociologique, Paris, Alcan, 1899.

Bouglé, Célestin, Qu'est-ce que la sociologie? Paris, Alcan, 1907.

Coenen-Huther, Jacques, Observation participante et théorie sociologique, Paris, L'Harmattan, 1995.

Coenen-Huther, Jacques, «Le type idéal comme instrument de la recherche sociologique», Revue française de sociologie, 44-3, juillet-septembre 2003, pp. 531-547.

Coenen-Huther, Jacques, Sociologie des élites, Paris, Armand Colin, 2004.

Coenen-Huther, Jacques, «La cumulativité du savoir sociologique entre mythe et réalité», Revue Européenne des Sciences Sociales, Tome XLIII, No 131, 2005a, pp. 23-33.

Coenen-Huther, Jacques, «Heurs et malheurs du concept de rôle social», Revue Européenne des Sciences Sociales, Tome XLIII, N 132, 2005b, pp. 65-82.

Coenen-Huther, Jacques, «Eugène Dupréel, philosophe, sociologue et moraliste », Revue Européenne des Sciences Sociales, Tome XLIV, N 134, 2006, pp. 97-118.

Davy, Georges, «Compte rendu de Sociologie générale par E. Dupréel», L'Année sociologique, troisième série, 1948-49, pp. 123-130.

Dupréel, Eugène, Le rapport social, Paris, Alcan, 1912.

Dupréel, Eugène, Sociologie générale, Paris, PUF, 1948

Dupréel, Eugène, Essais pluralistes, Paris, PUF, 1949.

Glaser, Barney G. et Strauss, Anselm L., The Discovery of Grounded Theory, Chicago, Aldine, 1967, 1973.

Glaser, Barney G. et Strauss, Anselm L., Awareness of Dying, Chicago, Aldine Publishing Company, 1965.

Hersch, Jeanne, L'étonnement philosophique, Paris, Gallimard, 1981; coll. «Folio/Essais», 1993.

Léger, François, La pensée de Georg Simmel, Paris, Editions Kimé, 1989.

Lévi-Strauss, Claude, «Introduction à l'œuvre de Marcel Mauss», in: Marcel Mauss, Sociologie et anthropologie, Paris, PUF, 1950.

Lévi-Strauss, Claude, Anthropologie structurale, Paris, Plon, 1958.

Lévi-Strauss, Claude, Anthropologie structurale deux, Paris, Plon, 1973.

Lévi-Strauss, Claude, Le regard éloigné, Paris, Plon, 1983.

Marx, Karl et Engels, Friedrich, L'idéologie allemande, 1846, traduit de l'allemand par H. Auger et al., Paris, Ed. Sociales, 1968.

Mendras, Henry et Forsé, Michel, Le changement social. Tendances et paradigmes, Paris, Armand Colin, 1983.

Monod, Jacques, Le hasard et la nécessité, Paris, Seuil, 1970.

Piaget, Jean, Logique et connaissance scientifique, Paris, Gallimard, Coll. «La Pléiade», 1967.

Simmel, Georg, Soziologie, Leipzig, Duncker und Humblot, 1908. Trad. fr. Sociologie. Etude sur les formes de la socialisation, Paris, PUF, 1999.

Simmel, Georg, Die Probleme der Geschichtsphilosophie, Leipzig, Duncker und Humblot, $5^{\mathrm{e}}$ édition, 1923. Trad. fr. par Raymond Boudon, Les problèmes de la philosophie de l'histoire, Paris, PUF, 1984.

Simmel, Georg, Grundfragen der Soziologie, 1917. Trad. fr. Sociologie et épistémologie, Paris, PUF, 1981.

Tarde, Gabriel, Les lois de l'imitation, Paris, Alcan, 1890. Quatrième édition revue et augmentée, 1904.

Vandenberghe, Frédéric, La sociologie de Georg Simmel, Paris, La Découverte, 2001.

Wiese, Ludwig von, System der allgemeinen Soziologie als Lehre von den sozialen Prozessen und den sozialen Gebilde der Menschen, Berlin, Duncker und Humblot, 1928. 